\title{
Impacto psicossocial na reabilitação estética anterior com resina composta direta: relato de caso
}

Psychosocial impact on anterior aesthetic rehabilitation with direct composite resin: case report

Impacto psicosocial en la rehabilitación estética anterior con resina compuesta directa: reporte de un caso

Matheus de Jesus BENTO ${ }^{1}$

Victor Augusto Alves BENTO2

Daisilene Baena CASTILLO ${ }^{1}$

Fabrícia Araújo PEREIRA

${ }^{1}$ Faculdade de Odontologia, Universidade Federal de Mato Grosso do Sul (FAODO/UFMS) 79070-900 Campo Grande - MS, Brasil

2Programa de Pós-Graduação em Odontologia, UNESP - Universidade Estadual Paulista "Júlio de Mesquita Filho",

Faculdade de Odontologia de Araçatuba, 16015-050 Araçatuba- SP, Brasil

\section{Resumo}

Devido a crescente valorização da harmonização facial, faz grande a procura de tratamentos estéticos nas clínicas odontológicas, devido aos fatores que interferem na harmonia do sorriso. Com isso, cada vez mais se torna um desafio satisfazer as exigências estéticas de cada paciente. Portanto, o objetivo deste trabalho foi relatar o impacto psicossocial em um caso clínico que foi realizado reabilitação estética anterior com resina composta direta. O paciente, gênero masculino, 32 an os, compareceu à clínica de Dentística da Faculdade de Odontologia da Universidade Federal de Mato Grosso do Sul (FAODOUFMS) em 2019, queixando-se da aparência do seu sorriso devido ao "espaço" que havia entre os dentes e "cor amarelada". No exame clínico detalhado foi verificado a presença de diastema entre os elementos 11 e 21 , devido a desproporção do tamanho dos dentes nessa região. Foi proposto, então, clareamento de consultório combinado com caseiro e realização de facetas diretas de resina composta nos elementos 11,12,13, 21, 22 e 23. Para isso, foi realizado fotografias, enceramento diagnóstico inicial e teste com mock-up. As facetas foram confeccionadas pela técnica estratificada com auxílio de guia de silicone para confecção da face palatina. Foi aplicado o questionário OHIP-14 antes e após o tratamento para analisar a eficácia do tratamento na qualidade de vida do paciente. Concluiu-se o tratamento baseado no clareamento dental seguido por facetas diretas com resina composta foi capaz promover uma melhor qualidade de vida.

Descritores: Diastema; Estética Dentária; Clareamento Dental; Facetas Dentárias.

\section{Abstract}

Due to the increasing appreciation of facial harmonization, there is a great demand for aesthetic treatments in dental clinics, due to the factors that interfere with the harmony of the smile. As a result, it becomes increasingly challenging to satisfy the aesthetic requirements of each patient. Therefore, the objective of this work was to report the psychosocial impact in a clinical case that was carried out prior aesthetic rehabilitation with direct composite resin. The male patient, 32 years old, attended the Dentistry clinic of the Faculty of Dentistry of the Federal University of Mato Grosso do Sul (FAODO-UFMS) in 2019, complaining about the appearance of his smile due to the "space" that was between the teeth and "yellowish color". In the detailed clinical examination, the presence of diastema between elements 11 and 21 was verified, due to the disproportion of the size of the teeth in this region. Therefore, it was proposed to lighten the office combined with a home office and make direct composite resin veneers on elements 11,12,13, 21, 22 and 23. For this, photographs, initial diagnostic waxing and mock-up testing were performed. The veneers were made using the stratified technique with the aid of a silicone guide for making the palatal face. The OHIP-14 questionnaire was applied before and after treatment to analyze the effectiveness of the treatment on the patient's quality of life. We concluded the treatment based on tooth whitening followed by direct veneers with composite resin was able to promote a better quality of life.

Descriptors: Diastema; Esthetics, Dental; Tooth Bleaching; Dental Veneers.

\section{Resumen}

Debido a la creciente apreciación de la armonización facial, existe una gran demanda de tratamientos estéticos en clínicas dentales, debido a los factores que interfieren con la armonía de la sonrisa. Como resultado, se vuelve cada vez más difícil satisfacer los requisitos estéticos de cada paciente. Por lo tanto, el objetivo de este trabajo fue informar el impacto psicosocial en un caso clínico que se realizó previa rehabilitación estética con resina compuesta directa. El paciente masculino, de 32 años, asistió a la clínica de Odontología de la Facultad de Odontología de la Universidad Federal de Mato Grosso do Sul (FAODO-UFMS) en 2019, quejándose de la aparición de su sonrisa debido al "espacio" que había entre los dientes y el "color amarillento". En el examen clínico detallado, se verificó la presencia de diastema entre los elementos 11 y 21 , debido a la desproporción del tamaño de los dientes en esta región. Por lo tanto, se propuso aligerar la oficina combinada con una oficina en el hogar y hacer carillas de resina compuesta directa en los elementos 11,12,13, 21, 22 y 23 . Para esto, se realizaron fotografías, encerado de diagnóstico inicial y pruebas de maquetas. Las carillas se hicieron utilizando la técnica estratificada con la ayuda de una guía de silicona para hacer la cara palatina. El cuestionario OHIP-14 se aplicó antes y después del tratamiento para analizar la efectividad del tratamiento sobre la calidad de vida del paciente. Concluimos que el tratamiento basado en blanqueamiento dental seguido de carillas directas con resina compuesta fue capaz de promover una mejor calidad de vida.

Descriptores: Diastema; Estética Dental; Blanqueamiento de Dientes; Coronas com Frente Estético.

\section{INTRODUÇÃO}

Devido a crescente valorização da harmonização facial, incluindo um sorriso esteticamente agradável, faz grande o número de pessoas que visam uma melhora na sua autoimagem ${ }^{1-3}$, buscando uma maior aceitação social por meio de um tratamento odontológico ${ }^{4,5}$. O tratamento adequado para correções de dentes com alteração de cor, forma e/ou tamanho depende da condição clínica do paciente, do conhecimento do operador, das técnicas disponíveis e dos materiais empregados ${ }^{2,6-9}$.

Um sorriso esteticamente agradável se dá pela harmonia simétrica dos dentes com a linha média facial, o que faz com que discrepâncias dentárias ou distribuições inadequadas dos espaçamentos dentários anteriores criem uma aparência desagradável 
tanto para os pacientes quanto para o observador $^{10}$. Assim, diastemas na região da linha média maxilar podem afetar a autoestima dos pacientes, sendo um fator negativo na autopercepção da estética dental ${ }^{11}$.

O Cirurgião-Dentista deve compreender a etiologia do diastema dental que pode estar relacionada a anomalia no número ou no tamanho dentário, à inserção do freio labial, a hábitos orais deletérios ou à periodontite avançada $^{11}$. Assim, conseguir estabelecer 0 correto tratamento dentro das variáveis opções: aparelhos ortodônticos, próteses fixas, laminados cerâmicos e facetas diretas com resina composta ${ }^{12}$. O profissional deve sempre preconizar pelo tratamento mais conservador, não pensando apenas em termos estéticos como também no excelente prognóstico em longo prazo ${ }^{13}$.

As facetas de técnica direta com resinas compostas se destacam como tratamento para fechamento de diastemas, considerado um procedimento minimamente invasivo, que fornece excelente previsibilidade de resultados satisfatório $^{13,14}$. Além da possibilidade da realização em sessão única, com baixo custo ao paciente, esse procedimento apresenta excelente potencial para devolver função de longa duração que emulam perfeitamente os tecidos dentários naturais, mesmo na área anterior ${ }^{10,12,15}$, principalmente pelo desenvolvimento destes materiais com diferentes graus de translucidez ${ }^{16}$.

Intervenções odontológicas podem causar variações no bem estar do paciente, no qual, sua satisfação com o resultado do tratamento interfere diretamente em seu contexto social ${ }^{17,18}$. Para mensurar o impacto de tais condições orais na qualidade de vida é possível utilizar o questionário OHIP-14 (Oral Health Impact Profile) ${ }^{19}$.

O propósito deste trabalho foi relatar 0 impacto psicossocial em um caso clínico de reabilitação estética anterior através das técnicas de clareamento dental e confecção de facetas com resina composta.

\section{CASO CLÍNICO}

Paciente, gênero masculino, 32 anos, compareceu à clínica de Dentística da Faculdade de Odontologia da Universidade Federal de Mato Grosso do Sul (FAODO-UFMS) em 2019, queixando-se da aparência do seu sorriso devido ao "espaço" que havia entre os dentes e "cor amarelada". Após anamnese e exame clínico detalhado foi verificada a presença de diastema entre os elementos $11 \mathrm{e}$ 21, devido a desproporção do tamanho dos dentes nessa região. Na primeira consulta, foram feitos exames clínicos, radiográficos, além de uma avaliação facial completa com fotografias intra e extraorais (Figuras 1 e 2), impressões com hidrocolóide irreversível alginato Jeltrate ® Plus (Dentsply - Petrópolis, RJ) e aplicação do questionário OHIP-14 a fim de avaliar o impacto das suas condições bucais atuais em sua qualidade de vida.

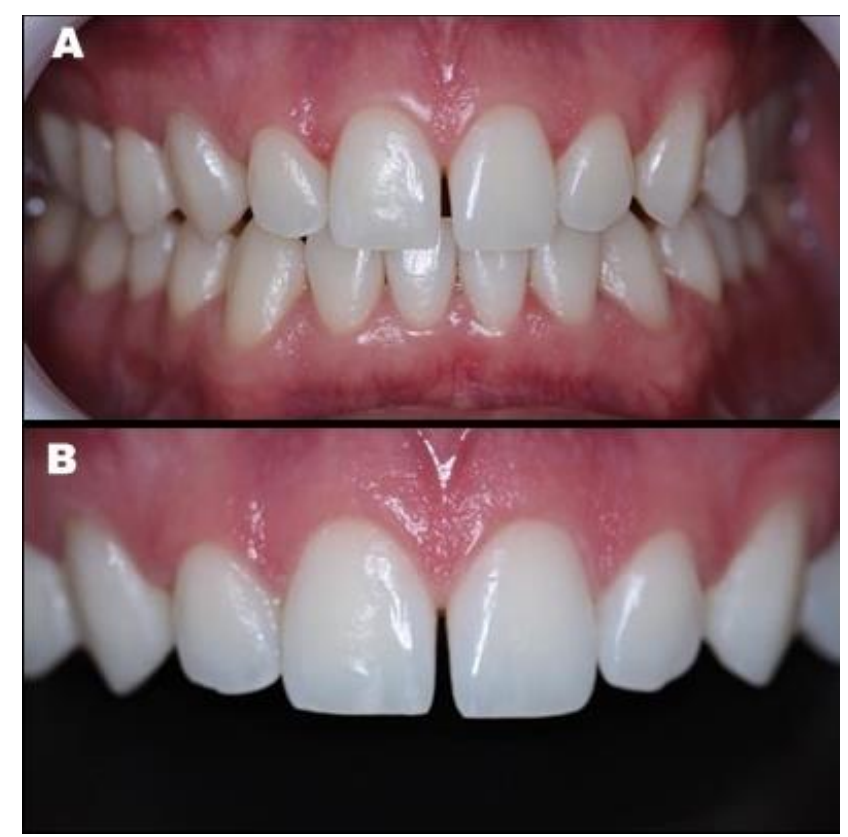

Figura 1: Fotografias intraorais para planejamento. A: Vista em oclusão. B: Vista dos incisivos superiores.

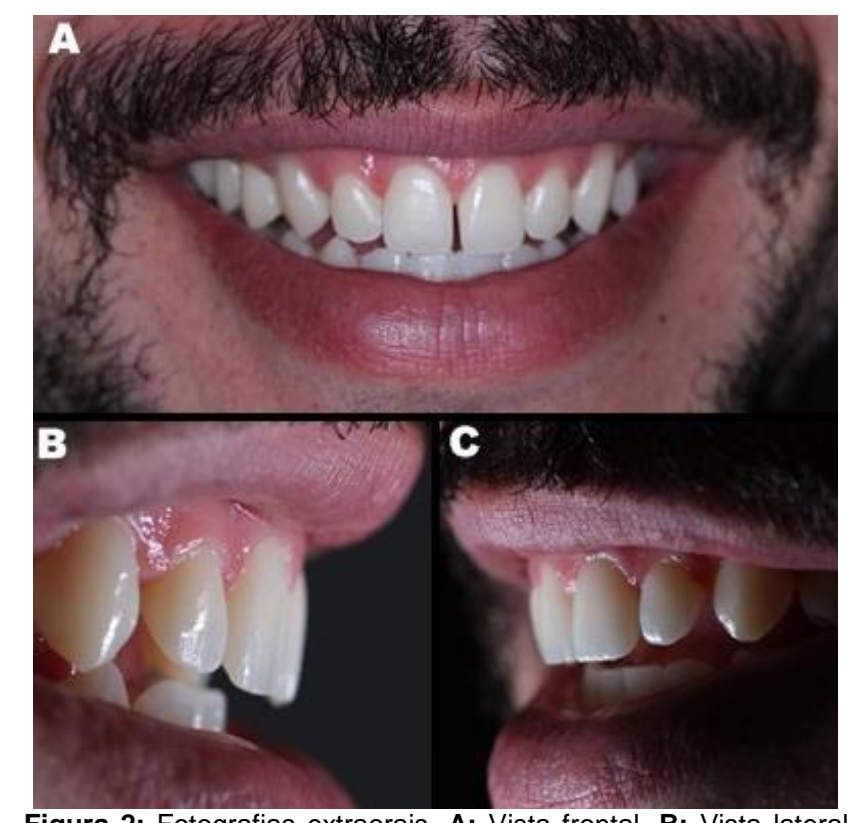

Figura 2: Fotografias extraorais. A: Vista frontal. B: Vista lateral direita. C: Vista lateral esquerda.

O planejamento do caso foi realizado por meio de fotografias e modelos de gesso, sendo analisado a forma, o tamanho e a posição dos dentes em ambas as arcadas. Os modelos de gesso foram utilizados para um enceramento de diagnóstico definindo o formato e tamanho dos dentes (Figura 3). Após, foi confeccionado uma guia de silicone de adição Virtual ${ }^{\circledR}$ (Ivoclair 
Vivadent - Barueri, SP) - pesada e leve para realizar um mock-up com resina bisacrílica Systemp C\&B® (Ivoclair Vivadent - Barueri, SP), com objetivo de simular o resultado estético final e obter a análise e aceitação do paciente (Figura 4).

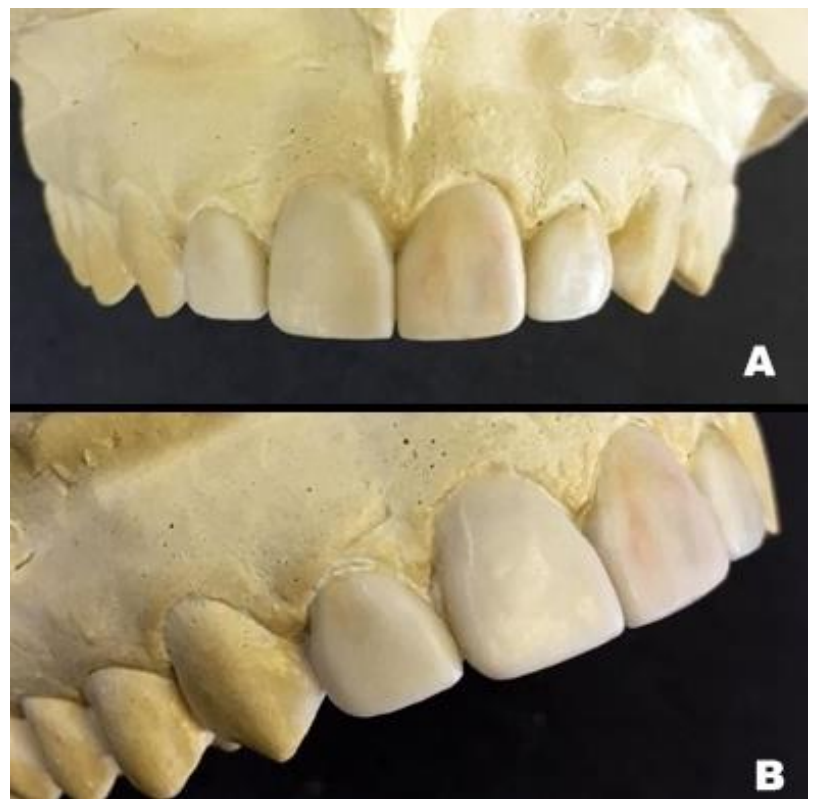

Figura 3: Enceramento de diagnóstico. A: Vista frontal. B: Vista lateral.

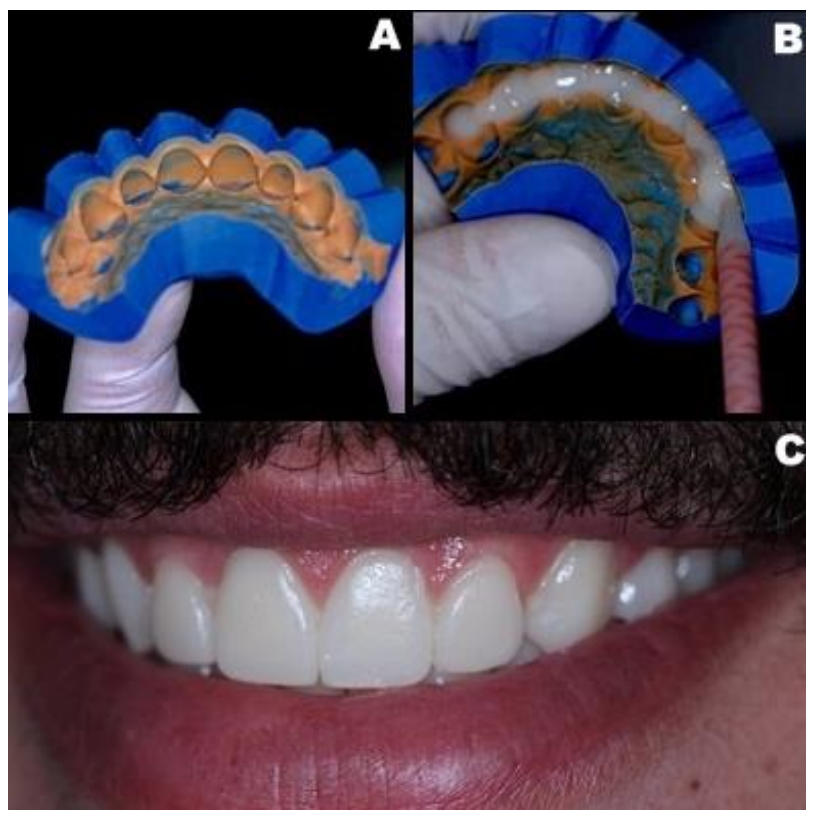

Figura 4: Teste com mock-up. A: Guia de silicone de adição. B: Aplicação da resina bisacrílica. C: Simulação do aspecto estético final.

O plano de tratamento envolveu clareamento dental de Técnica Combinada: uma sessão de consultório, uma semana de clareamento caseiro com moldeiras, e mais uma sessão em consultório. Depois, a realização de facetas diretas de resina composta nos elementos 11,12,13, 21, 22 e 23. A opção pelo tratamento com facetas diretas deu-se principalmente por não haver necessidade de desgaste dental.

$\mathrm{Na}$ primeira sessão de clareamento de consultório foi identificado, antes do procedimento, a cor A3, segundo a escala Vitta pan Classical. Utilizou-se peróxido de hidrogênio 35\% Whiteness HP-Blue ® (FGM Joinville, SC) com aplicação de 40 minutos e, ao final do procedimento, observou-se aumento do valor da cor, saltando de A3 para A2. Em seguida, foi entregue ao paciente moldeiras de clareamento placa flexível $133 \mathrm{~mm}$ Whiteness $\AA$ (FGM - Joinville, SC), obtido pelos modelos de gesso do paciente, e a seringa de peróxido de carbamida 22\% Whiteness Perfect ${ }^{\circledR}$ (FGM Joinville, SC). Foram dadas as devidas orientações quanto ao protocolo do clareamento caseiro. Com uma semana, a cor passou de A2 para $A 1$, e com a última sessão de clareamento de consultório chegou-se na cor B1. E então marcou-se a execução das facetas para após duas semanas.

Sob isolamento absoluto modificado e afastamento gengival com o posicionamento de fio afastador 000 Ultrapak $\AA$ (Ultradent Utah,USA), os elementos dentários receberam condicionamento ácido com ácido fosfórico 37\% em gel Acid Gel® (Villevie - Joinville, SC) por 30 segundos seguido de lavagem com jato de ar e água pelo mesmo tempo (Figura 5).

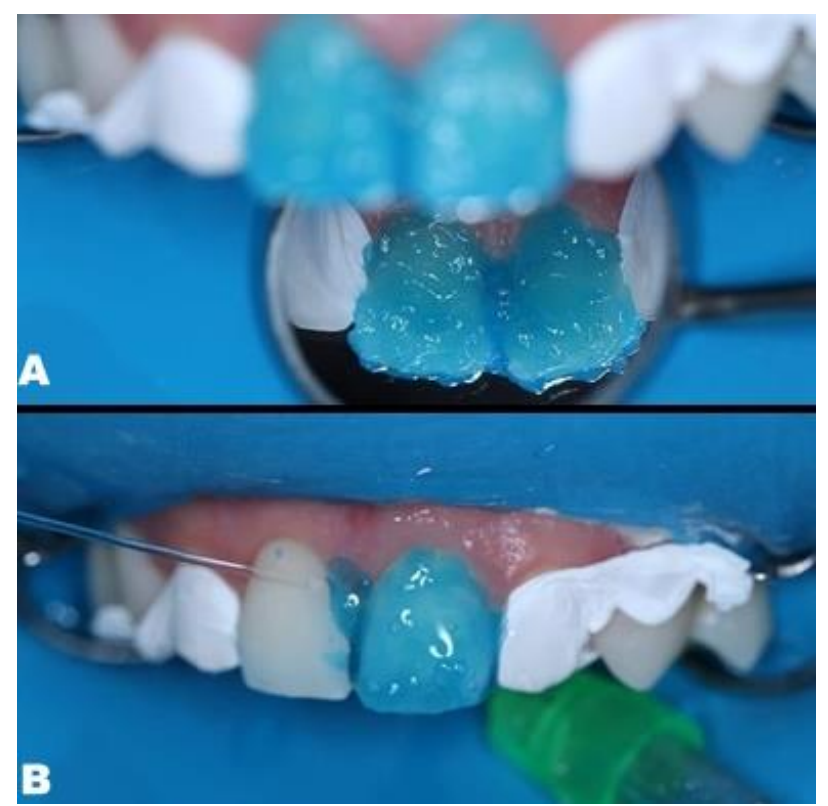

Figura 5: Condicionamento ácido. A: Aplicação ácido fosfórico 37\%. B: Lavagem.

O sistema adesivo de escolha foi o Scotch Bond Multi Purpose (3M - ESPE), onde foi aplicado somente o Bond, frasco número dois, com o auxílio de pincel Microbrush (KG) e posterior fotopolimerização por 20 segundos (Figura 6). As facetas foram confeccionadas pela técnica estratificada com a utilização das resinas composta nanoparticulada Filtek Z350® (3M ESPE - São Paulo, SP) de cores EB1 (esmalte) e B1B (corpo). As faces palatinas dos dentes foram reproduzidas com auxílio de uma 
guia de silicona de adição (3M ESPE Express STD), confeccionada a partir do enceramento diagnóstico. Após a construção do anteparo palatino com resina de esmalte, realizou-se os incrementos com resina de corpo e finalizou-se com resina de esmalte (Figura 7). Esta etapa incluiu aplicação de conceitos de macro e microestética, estratificação e anatomia dentária. Cada incremento foi fotopolimerizado por 30 segundos, com aparelho de Led Fotopolimerizador Emitter A Fit® (Schuster Santa Maria, RS).
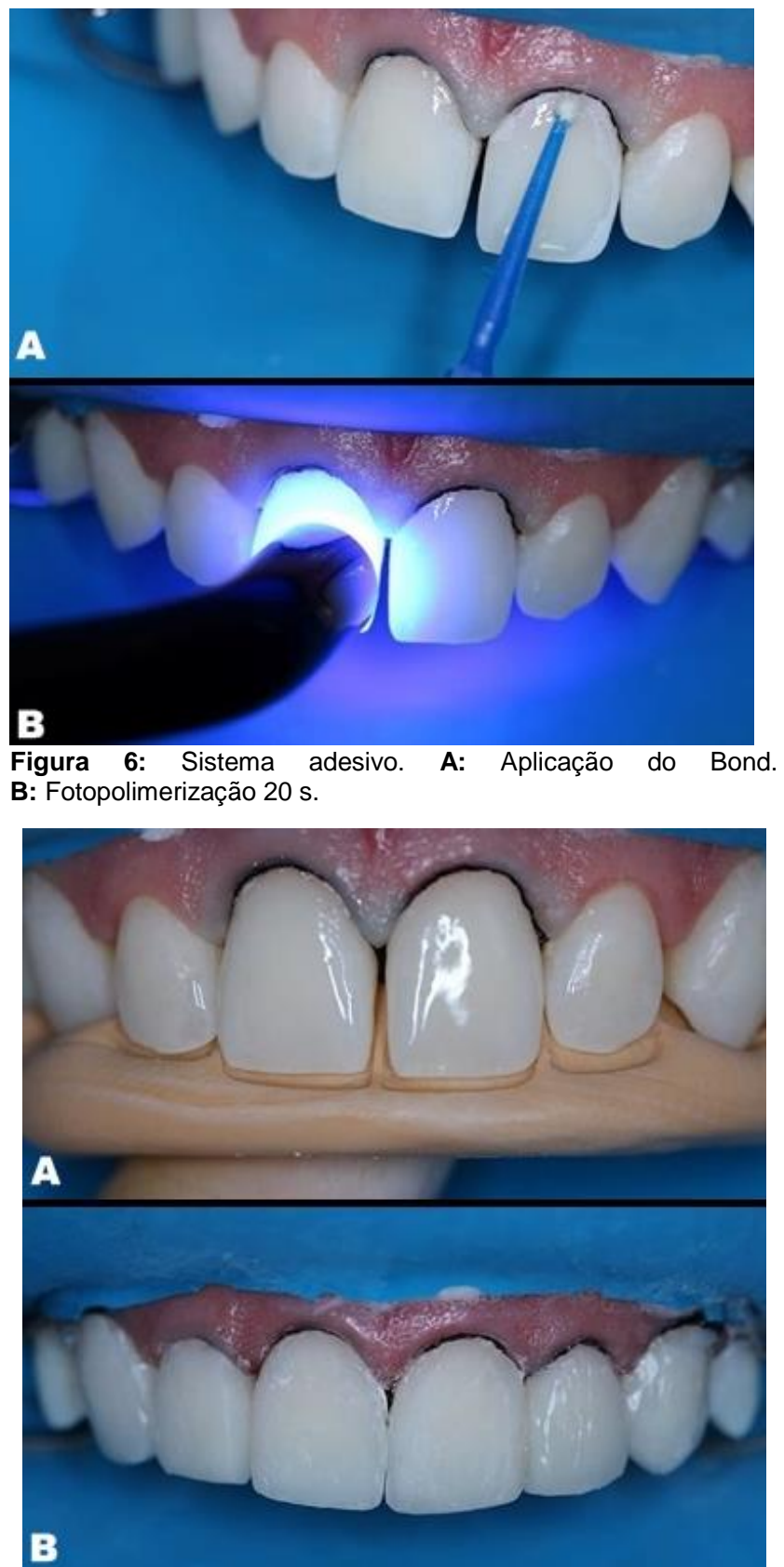

Figura 7: Técnica da estratificação. A: Construção da face palatina com auxílio da guia de silicone. B: Recobrimento das restaurações com última camada de resina esmalte.

O acabamento das restaurações foi feito utilizando-se lâminas de bisturi no 12 (SwannMorton), brocas de acabamento ponta $3195 \mathrm{~F}$ (KG Sorensen® - Cotia, SP) e discos de lixa Sof-Lex Pop On® (3M ESPE - São Paulo, SP).
Para a etapa de polimento, foram utilizadas um jogo de borrachas para polimento de resina Dhpro® (Dhpro - Paranaguá, PR) e disco de feltro associado à pasta de polimento Diamond Polish Mint ${ }^{\circledR}$ (Ultradent - Utah, USA). Ao final do procedimento obteve-se uma restauração em harmonia com sorriso do paciente (Figura 8). Após três semanas o paciente retornou e foi reaplicado o formulário OHIP-14 para avaliação do impacto do procedimento em sua qualidade de vida.

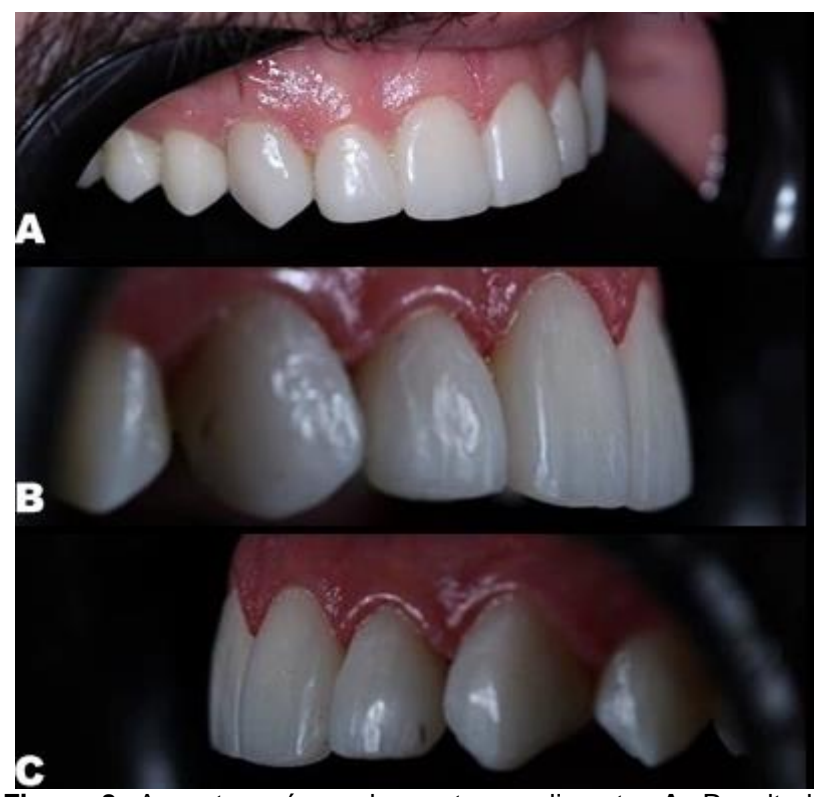

Figura 8: Aspecto após acabamento e polimento. A: Resultado final incisivos superiores. B: Vista lateral direita. C: Vista lateral esquerda.

Neste estudo, as pontuações do OHIP14 foram calculadas pelo método aditivo, sendo que maiores pontuações indicam uma qualidade de vida mais pobre ${ }^{20}$. Antes do tratamento o paciente apresentou uma pontuação de 24 pontos e após o tratamento a pontuação passou a ser de 5 pontos.

DISCUSSÃO

A avaliação da harmonia do sorriso é uma importante etapa no diagnóstico para tratamentos odontológicos estéticos ${ }^{3}$. No presente caso, o paciente apresentava diastema na região da linha média maxilar devido a desproporção de tamanho dos incisivos, exibindo uma assimetria e aspecto de sorriso infantil. Além disso, apresentava uma autopercepção de dentes "envelhecidos" devido ao aspecto amarelado.

No conceito atual da Odontologia restauradora, alterações antiestéticas de forma, posição e cor dos dentes podem ser solucionadas de maneira conservadora, sem a necessidade de preparos invasivos ou retenções adicionais, através da aplicação direta de resina composta ${ }^{21}$. Devido as propriedades específica deste material, frente a 
uma falha, torna-se possível a confecção de reparo sem a necessidade de substituição completa da restauração, assim aumentando sua longevidade e evitando 0 ciclo restaurador ${ }^{22}$, sendo esta a escolha para 0 presente caso. Em contrapartida, restaurações indiretas exigem um preparo dental mais extenso, não adaptando perfeitamente ao conceito de tratamento restaurador minimamente invasivo ${ }^{12}$.

De acordo com Prasada et al. $^{23}$, restaurações diretas em resina composta são preferíveis em casos de pequenos diastemas, apresentando um excelente resultado estético, durável e de ótimo custo benefício. Avaliações clínicas mostraram taxa de sobrevida de $89 \%$ de restaurações diretas de resina composta para fechamento de diastema em acompanhamento de cinco anos ${ }^{24,25}$. No estudo de Prabhu ett al. ${ }^{26}$, que obtiveram uma taxa de retenção de $91 \%$ das restaurações de diastema de linha média após um período de 5 anos. $O$ sucesso clínico deste material depende de seu correto manuseio e indicação adequada ${ }^{27}$.

De acordo com Alencar et al. ${ }^{28}$, os resultados da técnica restauradora direta podem ser aprimorados com clareamento dental prévio às restaurações. Após 0 término do clareamento, é necessário aguardar um tempo de dez a quinze dias, para que haja 0 estabelecimento final da cor e a remoção de oxigênio do interior dos tecidos dentários, para que o tratamento restaurador seja realizado, atingindo-se valores apropriados de resistência de união entre resina composta e esmalte dentário $^{29}$. No presente caso foi aguardado um período de 14 dias.

Um adequado diagnóstico e plano de tratamento são essenciais para um resultado de sucesso, sendo este influenciado pela expectativa do paciente em relação ao tratamento ${ }^{11}$. Neste estudo foi possível avaliar, através do questionário OHIP-14, o impacto psicossocial do tratamento na qualidade de vida do paciente, assim, confirmando sua satisfação.

CONCLUSÃO

A terapia estética de clareamento dental seguido pelas facetas anteriores para 0 fechamento do diastema na área da linha média maxilar e na harmonização do tamanho dos incisivos foi um tratamento efetivo, capaz de promover uma melhor qualidade de vida, contribuindo ao sucesso clínico.

\section{REFERÊNCIAS}

1. Cardenas AFM, Mora CAP, Siqueira FSF, Parreiras SO, Gomes JC. Restabelecimento estético de um sorriso envelhecido: Caso clínico. Rev APCD Estética. 2015;3(1):42-52.

2. Strasding M, Fehmer V, Pjetursson BE, Sailer I. Extending the service life of existing dental restorations with esthetic and functional limitations. J Prosthet Dent. 2018; 119(6):893-96.

3. Koidou VP, Rosenstiel SF, Rashid RG. Celebrity smile esthetics assessment: Smile angulation. J Prosthet Dent, 2017; 117(5):636-41.

4. Vellasco K, Campos I, Zouain-Ferreira TRF, Basting RT. Dentística minimamente invasiva: plástica dental. Arq Odontol. 2006; 42(2):104-12.

5. Simões MP, Albino LGB, Reis AF, Rodrigues JA. Restaurações estéticas conservadoras em dentes anteriores. R Dental Press Estét. 2009;6(1):90-101.

6. Levin, El. Dental esthetics and the golden proportion. J Prosthet Dent. 1978;40(3):244-52.

7. Flores-Mir C, Silva E, Barriga MI, Lagravere $\mathrm{MO}$, Major PW. Lay person's perception of smile aesthetics in dental and facial views. J Orthod. 2004;31(3):204-9.

8. Cotrim, ER, Vasconcelos Júnior, AV, Haddad, ACSS, Reis SAB. Perception of adults' smile esthetics among orthodontists, clinicians and laypeople. Dental Press J Orthod. 2015; 20(1):40-4.

9. Chaudhari A, Bagga DK, Agrawal P, Kalra H, Sirohi D. An assessment of the self-satisfying smile among different professionals. J Int Oral Health. 2018;10(3):111-14.

10. Hwang SK, Ha JH, Jin MU, Kim SK, Kim YK. Diastema closure using direct bonding restorations combined with orthodontic treatment: a case report. Restor Dent Endod. 2012;37(3):165-69.

11. Chu $\mathrm{CH}$, Zhang CF, Jin LJ. Treating a maxillary midline diastema in adult patients A general dentist's perspective. J Am Dent Assoc. 2011;142(11):1258-64.

12. Demirci M, Tuncer S, Öztas E, Tekçe N, Uysal Ö. A 4-year clinical evaluation of direct composite build-ups for space closure after orthodontic treatment. Clin Oral Investig. 2015;(9):2187-99.

13. Behle C. Placement of direct composite veneers utilizing a silicone buildup guide and intraoral mock-up. Pract Periodontics Aesthet Dent. 2000;12(3):259-66.

14. Xiaoling T, Ashraf MA, Yanyan Z. Paired observation on light-cured composite resin and nano-composite resin in den-tal caries repair. Pakistan J Pharma Sci. 2016; 29:2169-72.

15. Barrantes JCR, Araujo JrE, Baratieri LN. Clinical evaluation of direct composite resin restorations in fractured anterior teeth. ODOVTOS Int J Dent Sci. 2016; 16:47-61. 
16. Wolff D, Kraus T, Schach C, Pritsch M, Mente $\mathrm{J}$, Staehle HJ et al. Recontouring teeth and closing diastemas with direct composite buildups: A clinical evaluation of survival and quality parameters. J Dent. 2010; 38(12):1001-9.

17. Adulyanon S, Vourapukjaru J, Sheiham A. Oral impacts affecting daily performance in a low dental disease Thai population. Community Dent Oral Epidemiol. 1996;24(6):385-89.

18. Campos PRB, Amaral D, Silva MAC, Barreto SC, Pereira GDS. Reabilitação da estética na recuperação da harmonia do sorriso: relato de caso. RFO UPF. 2015;20(2):227-31.

19. Slade GD, Spencer AJ. Development and evaluation of the Oral Health Impact Profile. Community Dent Health. 1994;11(1):3-11.

20. Rosa FM, Hammershitt $T$, Zanchet $M$, Pozzobon R. A importância do enfoque multidisciplinar do recontorno estético de diastemas e incisivos conóides. Clín int j braz dent. 2007;3(1):42-8.

21. Frese C, Schiller P, Staehle HJ, Wolff D. Recontouring teeth and closing diastemas with direct composite buildups: a 5-year follow-up. J Dent. 2013;41(11):979-85.

22. Prasada G, Nakra O, Gowda M, Kumar A. Clinical Management of midline diastema. Indian J Dent Adv. 2014;6(1):1479-83.

23. Peumans $M$, Van Meerbeek $B$, Lambrechts $P$, Vanherle $\mathrm{G}$. The 5-year clinical performance of direct composite additions to correct tooth form and position. II. Marginal qualities. Clin Oral Investig. 1997;1(1):19-26.

24. Prabhu R, Bhaskaran S, Geetha Prabhu KR, Eswaran MA, Phanikrishna G, Deepthi B. Clinical evaluation of direct composite restoration done for midline diastema closure long-term study. J Pharm Bioallied Sci. 2015;7(Suppl 2):S559-62.

25. Wirsching E. Contemporary options for restoration of anterior teeth with composite. Quintessence Int. 2015;46(6):457-63.

26. Alencar MS, Araújo DFG, Maenosono RM, Ishikiriama BLC, Francischone CE, Ishikiriama SK. Reestablishment of esthetics with minimum thickness veneers: A one-year follow-up case report. Quintessence Int. 2014;45(7):593-97.

27. Lago ADN, Garone-Netto N. Microtensile bond strength of enamel after bleaching. Indian $\mathrm{J}$ Dent Res. 2013;24(1):104-9.

\section{CONFLITO DE INTERESSES}

Os autores declaram não haver conflitos de interesse

\section{AUTOR PARA CORRESPONDÊNCIA}

\section{Victor Augusto Alves Bento}

Departamento de Materiais Odontológicos e Prótese Faculdade de Odontologia de Araçatuba, UNESP

Rua José Bonifácio, 1193

16015-050 Araçatuba - SP, Brasil

E-mail: vtrbento97@gmail.com 